\title{
Chemical Anthropology: a study on the discrimination of human skeletons using x-ray fluorescence and chemometric tools
}

J. Gonzalez-Rodriguez* and G. Fowler

School of Life Sciences. University of Lincoln. Brayford Pool. Lincoln. LN6 7TS. United Kingdom. Tel. +441522886878 email: jgonzalezrodriguez@lincoln.ac.uk

\section{Abstract}

\section{Introduction}

On a global scale there are an increasing number of incidents where human remains are commingled and the need arises to reconcile these elements in order to identify the victims and return them to their families. Examples of where commingling occurs are natural disasters, transportation accidents and terrorist attacks such as that on the World Trade Centre in 2001. It is therefore imperative to have at hand a set of techniques to carry out a scientific process in which the correct remains are reunited with their families. Not only is this important to the dignity of the victims but also allows the families to begin the closure of the grieving process. In countries where armed conflict has taken place the victims of human rights abuses need to be identified and returned to their families once the appropriate analysis has taken place. However forensic anthropological investigations in countries where human rights violations have occurred are often restricted in their outcomes by the resources allocated to them, especially in terms of positively identifying the victims exhumed from commingled mass graves. Commingled mass graves can be defined as those graves that contain a number of disarticulated human remains from different individuals that have been mixed by either natural processes or human interventions. Commingled mass graves present a problem for anthropologists in that the remains of the same person need to be reconciled and returned to the family for burial. Re-associating skeletal elements requires a scientific approach especially in more complex cases where hundreds of parts are mixed together. The use of DNA analysis within the identification process is the principal method applied to this process, but can be constrained by a number of factors including the large cost of processing the samples, the condition of the bones, the availability of the families to supply DNA samples, and the existence of a family reference data base specific to that particular population. Traditional anthropological analysis, therefore, may not be enough to re-associate large numbers of skeletal elements and currently DNA remains the only option available to attempt this reconciliation.

$\mathrm{X}$-ray fluorescence spectrometry (XRF) is a technique that has been recently employed in both archaeological and forensic contexts. In archaeology it is useful to analyse trace element ratio in bone (9) and for provenance of archaeological artefacts (8). It has been introduced into the forensic domain in the search for trace evidence at crime scenes including gun shot residue and bodily fluids $(5,6)$ and been used to differentiate human from non-human material in cases of fragmented degraded samples (2). In a mass grave environment XRF could be used to analyse trace element ratios of individual bone elements with the aim of reconciling the parts to separate individuals. This 
has been attempted in the past using a destructive method and magnesium/zinc ratio was found to be the most reliable in separating elements (1).

This paper aims to apply the technique of non-destructive XRF analysis to test whether there is substantial differentiation within the trace elemental composition and their ratios of individuals to separate them using chemometric analysis. If successful this technique could be applied to mass grave and other situations such as mass disasters to reconcile elements without the use of expensive DNA analysis.

\section{Materials and methods}

A sample of 5 skeletons belonging to a medieval burial recently excavated within the city of Lincoln were selected for this study (Figure 1). These skeletons form part of a larger collection held at the University of Lincoln consisting of 47 individual inhumations and 163 skeletal elemental parts from St Katherines Priory. The site was excavated as part of a redevelopment of two Victorian properties by Pre-Construct archaeology within the monastic lands of St Katherine's Priory at the southern end of Lincoln High Street. The Priory was a mixed Gilbertine order which may explain the presence of female burials. The area forming the priory was extensive and included St Sepulcher's hospital where it is thought that the nuns cared for the sick and the monks were responsible for the maintenance and the every day running of the priory. The site excavated was used to inhume individuals from the mid $12^{\text {th }}$ to early $16^{\text {th }}$ century and is thought to form part of a cemetery within the Priory. Some of the individuals inhumed were probably wealthy and could afford the ostentation of a stone cist burial. Towards the end of the medieval period the cemetery was used for individuals belonging to a lower status who as a consequence were buried directly in the ground, probably wrapped in a shroud, which was common to this period.

Five adult articulated skeletons were selected for this study with over $95 \%$ of the skeletal elements present. The skeletons were inhumed in stone lined cists forming discrete burials therefore eliminating the possibility of commingling of parts. In order to test the hypothesis that individual bones belong to the same skeleton in terms of their elemental analysis, it was important that each skeleton selected was not commingled. The 5 skeletons consist of 4 males and 1 female with each only missing only a few hand and foot bones. The skeletons were cleaned using water and small brushes and left to dry naturally in the laboratory. Sex was determined using the skull and pelvis according to standard methods outlined in (4).

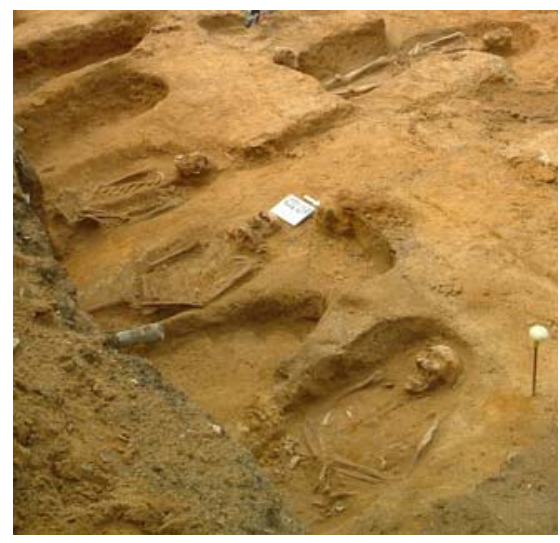


Figure 1 Part of the excavation at 45-47 St Catherines, Lincoln

A total of 23 bones from each individual were selected for this study; cranium (frontal), mandible (left and right rami), scapulae, clavicles, ribs (2), vertebrae (4), sacrum, ilium, metacarpus, femora and tibiae. Where the bone was paired both sides were sampled. In the case of the ribs, depending on the bone the flattest part of the bone was sought to take the measurement and repeated three times at the same site. The mean of the three measurements was calculated for that bone for each individual.

Multivariate Analysis was performed using the open source Tanagra software developed in the University Of Lyon (France) (Rakotomalala, 2005).

A portable X-Ray Fluorescence Niton XL3t Spectrometer analyser from Thermo Scientific (UK) was used for the elemental analysis.

\section{Results and discussion}

The analysis of the skeletal collection by X-Ray Fluorescence (XRF) began with the analysis of two individuals, a male and a female known as SK-45 and SK-3 respectively, in order to separate them. Using the elemental analysis information obtained from the XRF, a set of elements with biological, dietary or physiological significance were chosen, namely $\mathrm{Pb}, \mathrm{Sr}, \mathrm{Zn}, \mathrm{Fe}, \mathrm{Ca}$ and $\mathrm{K}$. Calcium forms part of the mineral content of the bone as hydroxylapatite $\left(\mathrm{Ca}_{10}\left(\mathrm{PO}_{4}\right)_{6}(\mathrm{OH})_{2}\right)$, which means that the different bone contents in this mineral can be traced through this element. Strontium is a very interesting mineral as, due to its chemical analogy, can replace $\mathrm{Ca}^{+2}$ very easily and its content in the bone is associated to diet. Zinc has also been considered by some authors as a paleodietary indicator (Gilbert, 1975; Ezzo, 1994) although this has not been thoroughly accepted (Weyder-Homeyer, 1993; Burton et al, 1999). Lead can also easily replace calcium in the bone and its levels in bone have been associated to exposure to one or more anthropogenic sources. Iron and potassium remain in the more amorphous mineral matrix in contact with blood vessels as they have their origin in the original blood content of the bone.

These elements were analysed and quantitated in the bones by XRF and the results exported to excel. The different bones analysed presented different levels of the elements of interest, which were used as raw data for analysis using Principal Component Analysis (PCA) and the results subjected to cross validation using Linear Discriminant Analysis (LDA) and the leave-one-out algorithm. The hypothesis to be proven is that different individuals will present unique levels of these elements in the bone depending on their own diet, physiology and metabolism. These elemental differences in the bones will be sufficient to separate the different individuals using multivariate analysis and the chemical composition of the different bones from the same skeleton will be similar enough to cluster them together.

The results of the PCA analysis can be seen in figure 1. There is a clear separation of the bones for two individuals based on their elemental concentration. The factor loadings and the correlation scatterplots for the analysis identified Pb, Sr and Fe (for PC1) and $\mathrm{Ca}$ and K (for PC2) as the most important variables influencing the separation. From this analysis we can infer that there is enough chemical difference to separate both skeletons but enough similarities to group them in the right group as the cross validation demonstrated with a $100 \%$ correct classification. 
The next step led to the use of three skeletons (SK45, SK4 (males) and SK3 (female)) to assess whether the differences in the chemical composition were still enough to correctly separate them. When the data were used to feed the PCA analysis under the supervised mode, the cross validation error rate using Linear Discriminant Analysis (LDA) indicated 100\% correct classification for SK-3 and 95.65\% for both SK-4 and SK-45 and a clear separation in the PCA.

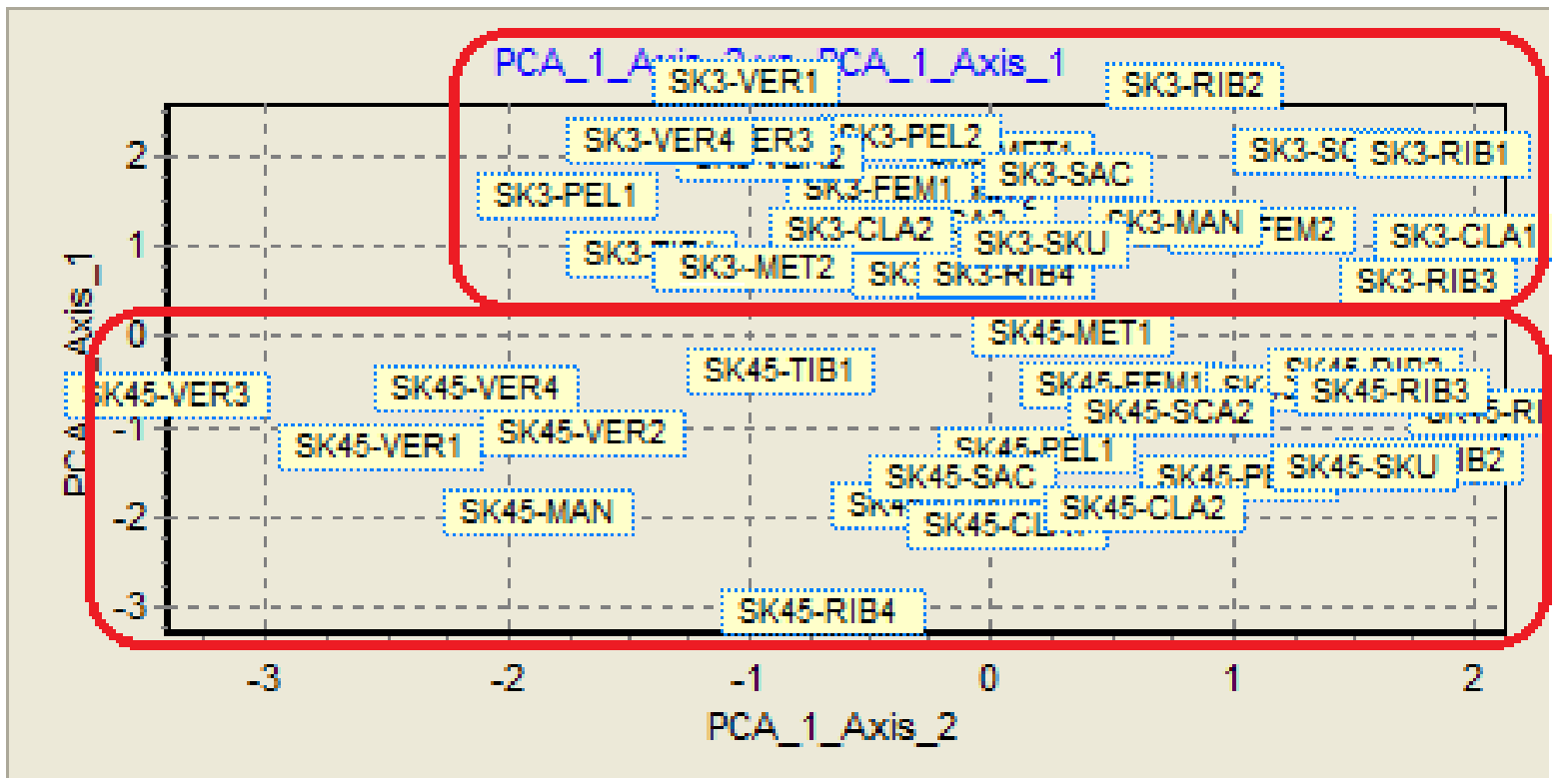

Figure 1. Principal Component Analysis of two skeletons from the sample set.

Although the separation obtained in the analysis was good, an indication that chemical noise could be affecting the separation was obtained. A way to improve the analysis was to use elemental ratios. The use of ratios has a double advantage: first it normalises the set of data avoiding the instrumental fluctuations in the analysis and second reduces the effect of the chemical background noise in the different analysis.

The use of ratios has been used in anthropology before to assess the dietary influence in different species and to assess the carnivorous or vegetarian status of the bone remains (Elias et al, 1982). In this sense the $\mathrm{Sr} / \mathrm{Ca}$ ratio was used for this task. Another ratio used to assess the influence of the lead incorporated into bones and the replacement by the $\mathrm{Pb}^{+2}$ ions in the bone structure is $\mathrm{Pb} / \mathrm{Ca}$. Following this trend of using elemental ratios with some significance, three new ratios were incorporated to the model. The $\mathrm{Sr} / \mathrm{Pb}$ ratio measured the competition between these two very similar ions aiming to replace calcium in the bone. This ratio can differ amongst individuals depending on the amount of Strontium and lead present in the environment and the different absorption rates for each individual in life. The next ratio implemented was $\mathrm{Zn} / \mathrm{Fe}$. As an average the human body contains between 2-3 grams of $\mathrm{Zn}$ stored in muscle and bones. $\mathrm{Zn}$ regulates more than 100 enzymes in the body so the amount present can greatly vary between individuals, for example, zinc concentration is greater in males than females. Iron is present in blood and on average a human body contains between 4-5 grams of which around 2.5 grams are in cells and the rest is stored as ferritin in the bone marrow. These two metals are strongly related to metabolic activities of the individual and its ratio will be related to metabolic activities, which is likely to be different between individuals. The last new ratio used is $\mathrm{K} / \mathrm{Fe}$. These two elements are present in blood in high 
concentration and are not directly stored in the bones, but trapped in the blood vessels irrigating the bone. Depending on the blood flow into the bone, which depends on the physiology of the individual and health status, this ratio will be different from individual to individual.

The same three skeletons used in the previous analysis were used to assess the use of ratios as raw data for the PCA analysis instead of single elements. The results of the PCA plot can be seen in Figure 2.

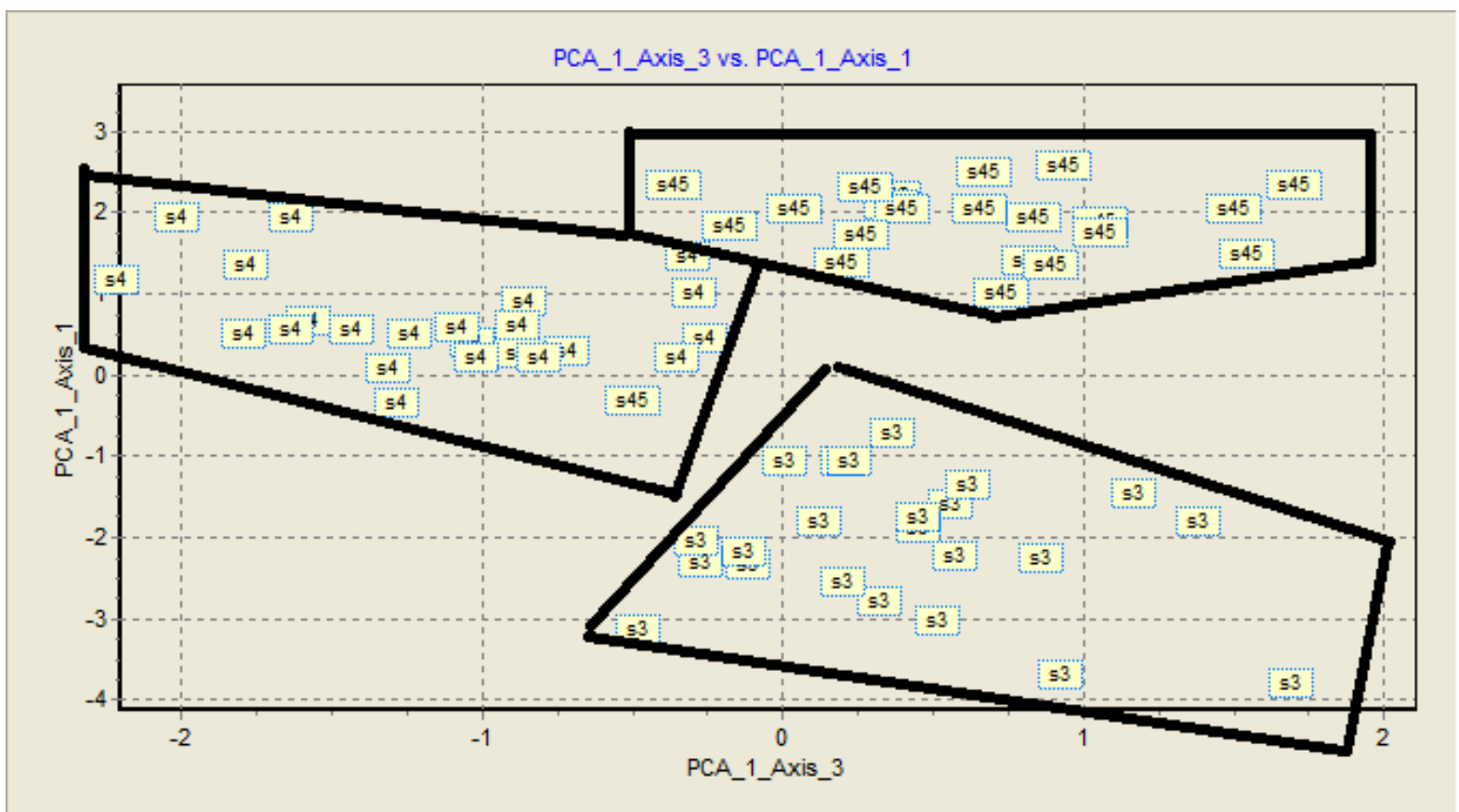

Figure 2. Principal Component Analysis of three skeletons from the sample set using elemental ratios: $\mathrm{Sr} / \mathrm{Ca}, \mathrm{Pb} / \mathrm{Ca}, \mathrm{Sr} / \mathrm{Pb}, \mathrm{Zn} / \mathrm{Fe}$ and $\mathrm{K} / \mathrm{Fe}$.

PC1 and PC3 showed the most discrimination power in the clustering of the different groups and were selected for the final analysis. When the supervised learning using LDA and the leave-one-out algorithm in the cross validation was performed, all samples were correctly classified using the ratios as compared to the previous analysis. The correlation scatterplot and the factor loadings showed that the least relevant of all the ratios was $\mathrm{Sr} / \mathrm{Ca}$ and was not used in the classification model this time. The rest of the elemental ratios presented similar levels of discrimination significance in PC1, between $73 \%$ and $81 \%$. The most influential ratios were the $\mathrm{Pb} / \mathrm{Ca}, \mathrm{Zn} / \mathrm{Fe}$ and $\mathrm{Sr} / \mathrm{Pb}$.

For the next analysis only the suggested ratios were used. The discrimination of four skeletons can be observed in figure 3 . In this case the cross validation analysis showed two of the skeletons with a 95.6\% correct classification and the other two showing $82.6 \%$ correct classification. As observed in the previous analysis, the $\mathrm{Sr} / \mathrm{Ca}$ ratio was the least significant variable influencing the separation model although this time was used to produce the separation as can be seen in the scatterplot in figure 4, and also as obtained in the factor loading data. The multivariate analysis still presented a good discrimination power, but some loss can be associated to the increase of the chemical background noise due to soil influence and diagenetic processes. The statistical noise observed in the multivariate analysis has a chemical explanation though. As the number of skeletons increases so 
does the chances of an increased contamination in the bones due to some elements present in the environment. Contamination coming from external sources affecting all the bones in the burial can alter their natural similarities and differences and making it easier to alter the classification. This process is occurring naturally in bones buried in the same area as the chemical composition of the soil is similar. In the case of our samples, the skeletons were buried individually but close enough to each other to assume the same soil chemical composition. The process of diagenesis, in which some minerals can be incorporated into the structure of the bone, is a slow process. Considering that, in our case, the skeletons are 500 to 800 years old this phenomenon plays an important role as many elements present in the soil were found to have perfused (permeated?) into the structure of the bone. Although the bones were cleaned before analysis, as explained in the materials and methods section, the presence of elements such as zirconium, titanium and manganese confirmed this contamination coming from the soil as these elements do not appear naturally in bone material. The average concentrations obtained from all the samples used in the study were $\mathrm{Mn}=354.3 \mathrm{mg} / \mathrm{L}$ with a $\% R S D=55.8, Z r=36.0 \mathrm{mg} / \mathrm{L}$ with a $\% R S D=20.9$ and $\mathrm{Ti}=365.5 \mathrm{mg} / \mathrm{L}$ with a $\% \mathrm{RSD}=12.2$. As it can be seen by the relative standard deviation (\%RSD) there is a high dispersion of the results which indicates that the absorption of the different elements by the bone is not even in all cases, with the exception of Titanium for which a relatively low \%RSD was obtained. This clearly can affect the clustering.

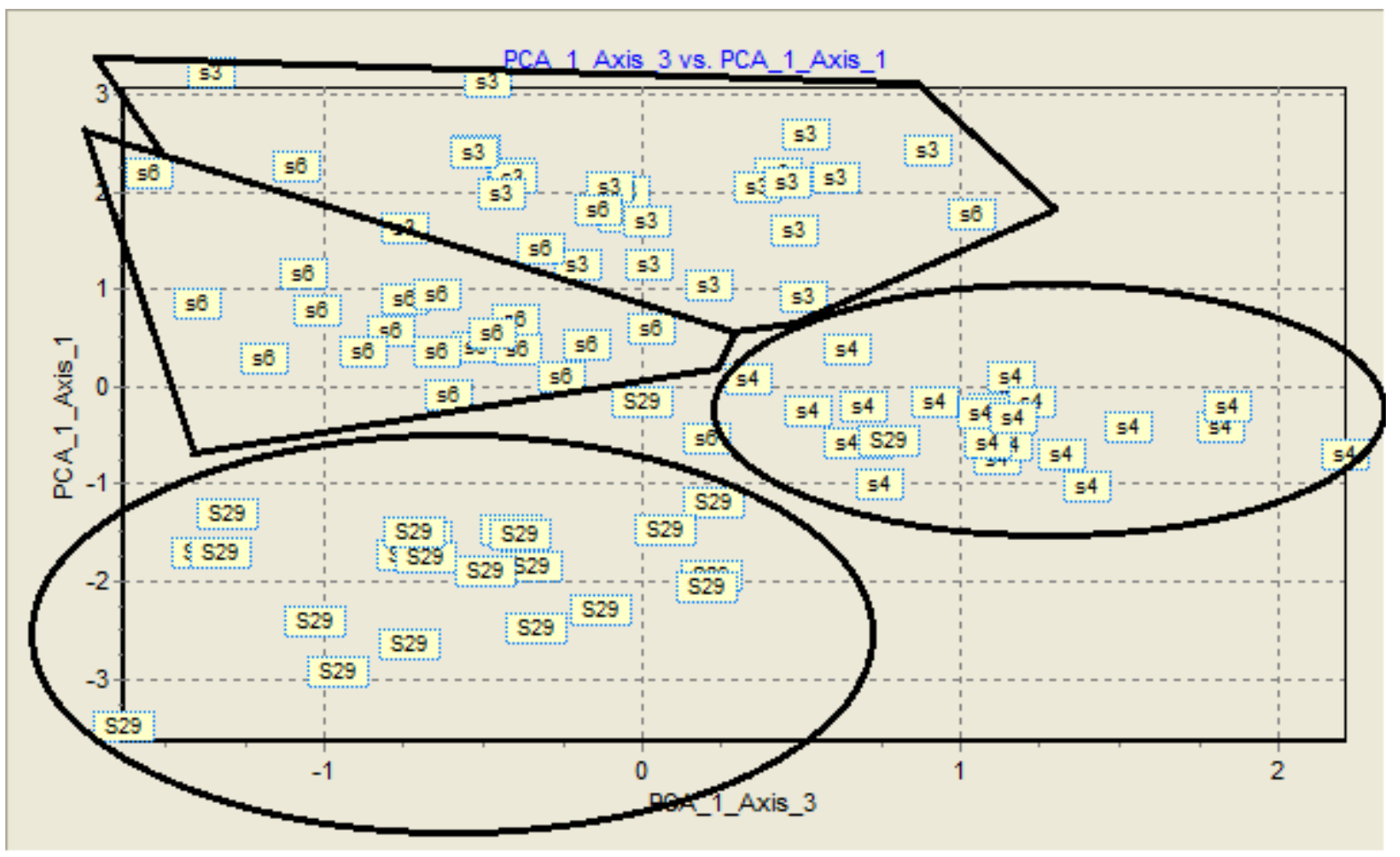

Figure 3. Principal Component Analysis of four skeletons from the sample set using elemental ratios: $\mathrm{Sr} / \mathrm{Ca}, \mathrm{Pb} / \mathrm{Ca}, \mathrm{Sr} / \mathrm{Pb}, \mathrm{Zn} / \mathrm{Fe}$ and $\mathrm{K} / \mathrm{Fe}$.

The observed uneven concentration of manganese and zirconium found in the different bones will greatly affect the chemical noise added to the PCA and may become an increasing problem when the number of individuals to be separated increases as it will increase the statistical noise affecting clustering. The age of the bones used in the study certainly plays a role as diagenesis is dependent 
on the time the bones are in contact with the soil or contaminants. The age of the bones is a factor to consider as it will certainly makes the separation more complicated, but still the combination of elemental ratios together with the use of chemometrics has proven effective to successfully separate three skeletons and still discriminated four with a very high degree of certainty.

Another suspected potential source of error in the acquisition of the data was the lack of homogeneity observed in the bones and their irregular shape. It is known that the distance from the object of study to the detector affects the intensity of the signal due to oxygen quenching. As not all the bones were flat and presented irregularities small differences in these distances may have played a role in increasing the experimental error and affected the quality of the raw data for the statistical analysis. In order to assess this, a metal alloy with known certified values was used to assess how distance could affect the analyses and what distances could be acceptable to avoid experimental error. The analysis point of the analyser was moved away sequentially from the object of analysis and the readings recorded at 1, 2, 3 and $4 \mathrm{~mm}$. The \%RSD obtained for all the measurements when compared to that obtained at $0 \mathrm{~mm}$ were in all cases lower than $3 \%$ demonstrating that in those cases in which the shape of the bone did not allow close contact with the head of the analyser a $4 \mathrm{~mm}$ distance would still provide accurate readings. This was the case for all bones analysed.

The analysis of five skeletons using the same methodological procedure demonstrated that, as expected, an increasing number of samples in which diagenetic processes have widely occurred might decrease the accuracy of the classification. The cross validation of the model using the leaveone-out algorithm yielded uneven results with the first skeleton being correctly classified at $95.6 \%$, second at $78.3 \%$, third at $73.9 \%$, fourth at $69.7 \%$ and fifth at $52.5 \%$. Still a clear separation for most bones from the different individuals can be achieved but, as predicted, the accuracy of the separation diminished and misclassification increased. Similar to the previous analyses, the ratio $\mathrm{Sr} / \mathrm{Ca}$ showed the lower contribution to the overall model when analysed using the correlation scatterplots and this was also supported by the factor loading analysis. As previously the most influential ratios were the $\mathrm{Pb} / \mathrm{Ca}, \mathrm{Zn} / \mathrm{Fe}$ and $\mathrm{Sr} / \mathrm{Pb}$ in $\mathrm{PC1}$. 


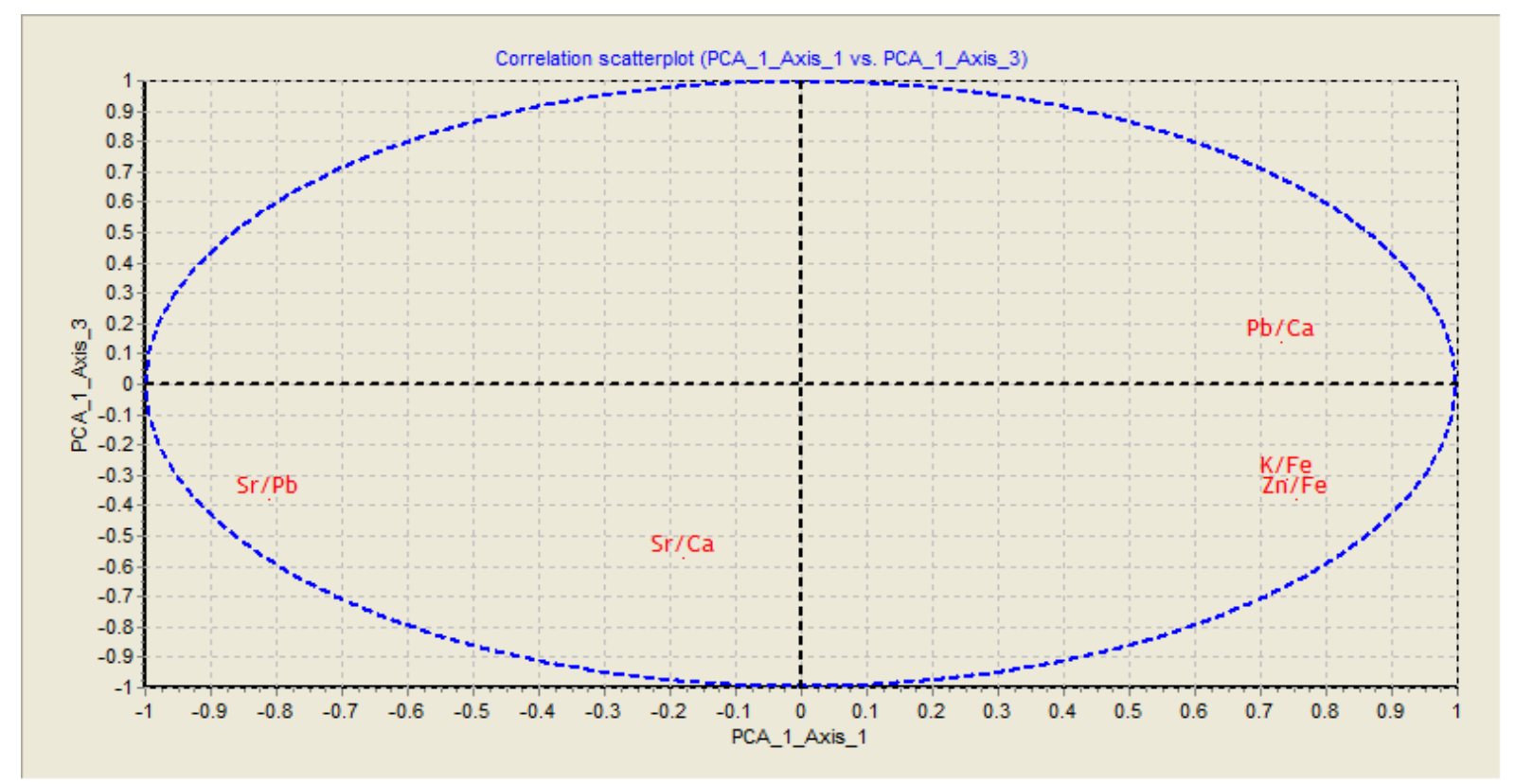

Figure 4. Correlation scatterplot for the separation of four skeletons using the elemental ratios: $\mathrm{Sr} / \mathrm{Ca}, \mathrm{Pb} / \mathrm{Ca}, \mathrm{Sr} / \mathrm{Pb}, \mathrm{Zn} / \mathrm{Fe}$ and $\mathrm{K} / \mathrm{Fe}$.

\section{Conclusion}

The results of the different atomic spectroscopic analyses combined with the use of multivariate analysis on a set of 5 skeletons produced a series of plots using Principal Component Analysis that helped to separate them with a high percentage of accuracy when two, three or four skeletons needed to be separated as demonstrated by the cross validation tools. A high degree of separation was achieved when five skeletons were used but the results showed a greater degree of dispersion and lower classification percentages.

The advantages of the use of X-Ray Fluorescence for the separation of human remains obtained from commingled mass graves are evident: XRF is portable and can be taken to remote areas if needed; it is a relatively inexpensive technique in comparison to DNA analysis and there is no need for a expensively-equipped laboratory or chemicals to develop the results; analyses can be performed in 30 seconds and an identification and separation can be achieved within hours; most importantly this is a non-destructive technique and the remains can be returned to the families intact. In terms of archaeological specimens it is important to preserve the skeletons and use nondestructive techniques so they can be preserved for future generations to study.

These advantages present this technique as the ideal screening technique prior to DNA analysis for confirmation of identification and present a feasible alternative in field work or when combined with DNA analysis can accelerate the identification of human remains as only those bones in doubt will need to be analysed by DNA techniques speeding up the whole process. 